\title{
Articulando Combinatória e Probabilidade: Jovens e Adultos Revisitando Problemas Combinatórios via Construção de Espaços Amostrais
}

\author{
Ewellen Tenorio de Lima \\ ewellentlima@gmail.com \\ https://orcid.org/0000-0002-3654-0370 \\ Universidade Federal de Pernambuco (UFPE) \\ Recife, Brasil. \\ Rute Elizabete de Souza Rosa Borba \\ resrborba@gmail.com \\ https://orcid.org/0000-0002-5098-4461 \\ Universidade Federal de Pernambuco (UFPE) \\ Recife, Brasil.
}

Recebido: 22/09/2020 Aceptado: 19/02/2021

\begin{abstract}
Resumo
O presente artigo discute contribuições que a articulação entre combinatória e probabilidade baseada na construção de espaços amostrais pode trazer para o desempenho na resolução de problemas combinatórios variados (arranjo, combinação, permutação e produto cartesiano). Os dados da pesquisa foram coletados junto a 24 estudantes brasileiros, concluintes de diferentes etapas da escolarização básica na modalidade de ensino denominada Educação de Jovens e Adultos. Corroborando achados de estudos anteriores, o problema de produto cartesiano foi aquele no qual os melhores desempenhos foram observados, enquanto o de combinação foi aquele no qual os desempenhos mais fracos foram obtidos pelos participantes. A revisitação aos problemas propostos, nos dois tipos de teste utilizados, consistiu em um rico momento de descoberta de novas possibilidades e/ou análise daquelas já indicadas. Tal articulação, pautada em revisitações, proporcionou, ainda, uma oportunidade de reflexões acerca das relações de ordem e de escolha que caracterizam cada uma das situações combinatórias exploradas nos problemas. Estes resultados dão indícios da potencialidade de um trabalho articulado entre combinatória e probabilidade para o desenvolvimento de ambos os raciocínios.
\end{abstract}

Palavras chave: Combinatória. Probabilidade. Educação de Jovens e Adultos. Espaço Amostral.

\section{Articulando Combinatoria y Probabilidad: Jóvenes y Adultos Revisitando Problemas Combinatorios via Construcción de Espacios Muestrales}

\section{Resumen}

En el presente artículo se analizan las contribuciones que la articulación entre combinatoria y probabilidad, basada en la construcción de espacios muestrales, puede aportar al desempeño en la resolución de problemas combinatorios variados (variación, combinación, permutación y producto cartesiano). Los datos de la investigación fueron colectados de 24 estudiantes brasileños, en diferentes etapas de la educación básica en la modalidad de enseñanza llamada Educación de Jóvenes y Adultos. Corroborando hallazgos de estudios previos, el problema del producto cartesiano fue aquel en el que se observaron los mejores desempeños, mientras que el problema de combinación fue aquel en el que los participantes obtuvieron los desempeños más débiles. La revisión de los problemas propostos, en los dos tipos de de pruebas utilizadas, 
consistió en un momento rico de descubrimiento de nuevas posibilidades y análisis de las ya señaladas. Dicha articulación, guiada por las revisiones, brindó también la oportunidad de reflexionar sobre las relaciones de orden y elección que caracterizan cada una de las situaciones combinatorias exploradas en los problemas. Estos resultados evidencian el potencial de un trabajo articulado entre combinatoria y probabilidad para el desarrollo de ambos razonamientos.

Palabras clave: Combinatoria. Probabilidad. Educación de Jóvenes y Adultos. Espacios Muestrales.

\title{
Articulating Combinatorics and Probability: Young People and Adults Revisiting Combinatorial Problems via Construction of Sample Spaces
}

\begin{abstract}
The current article discusses contributions that the articulation between combinatorics and probability, based on the construction of sample spaces, can bring to the performance in the resolution of varied combinatorial problems (arrangements, combinations, permutations and cartesian products). The research data were collected from 24 Brazilian students of youth and adult education in different stages of schooling. Corroborating findings of previous studies, the cartesian product problem was the one in which the best performances were observed, while the combination problem was the one in which the weakest performances were obtained by the participants. Revisiting these problems, in both the tests used, consisted in a rich moment of discovering new possibilities as well as analysing those already indicated. Such articulation, guided by revisitations, also provided an opportunity for reflections on the relations of order and choice - that characterise and distinguish each of the combinatorial situations explored in the problems. These results give evidences of the potential of an articulated work-between combinatorics and probability aiming at the development of both types of reasoning.
\end{abstract}

Keywords: Combinatorics. Probability. Youth and Adult Education. Sample Space.

\section{Introdução}

No Brasil instituiu-se oficialmente a oferta gratuita de educação básica, em seus diferentes níveis, voltada a jovens e adultos que, por motivos diversos, não conseguiram concluir seus estudos em idade regular. No texto da Lei de Diretrizes e Bases da Educação Nacional - LDB, no 9.394 (MEC, 1996), é ressaltado que a Educação de Jovens e Adultos (EJA) deve ser organizada e ofertada de modo que seja adequada ao seu público alvo, isto é, que se considerem suas especificidades, visto que a realidade, vivências e experiências do estudante jovem e adulto são distintas daquelas das crianças e adolescentes que cursam a educação básica no ensino regular.

Nesse sentido, para que seja possível ofertar educação adequada às necessidades e características particulares de estudantes jovens e adultos, é de suma importância que os conhecimentos e dificuldades de tais estudantes referentes a diferentes áreas sejam alvo de 
pesquisas, dado que no ambiente escolar, no qual os estudantes da EJA são (re)inseridos, “currículos, programas e métodos de ensino foram originalmente concebidos para crianças e adolescentes que percorreriam o caminho da escolaridade de forma regular" (Oliveira, 1999, p. 61). Assim, é por meio da realização de pesquisas, isto é, a partir da sondagem de conhecimentos de grupos de estudantes, que é possível conhecer fragilidades na aprendizagem de determinados conceitos, bem como reconhecer conhecimentos prévios desses estudantes (jovens e adultos) adquiridos dentro e fora da sala de aula.

A pesquisa da qual o presente texto apresenta um recorte (Lima, 2018), buscou trazer contribuições nesse sentido, tendo estudantes jovens e adultos como alvo para o desenvolvimento de um estudo de sondagem de conhecimentos de combinatória e de probabilidade mobilizados por tais estudantes em contexto de resolução de problemas, com uma proposta de articulação entre as duas áreas. Buscou-se investigar, especificamente, as relações que se estabelecem nesse contexto entre o raciocínio combinatório e o raciocínio probabilístico de estudantes jovens e adultos, explorando-se diferentes situações, suas características e relações invariantes e a partir do uso de representações simbólicas variadas, considerando-se, assim, o tripé conceitual defendido por Vergnaud $(1986,1996)$ como principal aporte teórico.

A escolha do foco da pesquisa - combinatória, probabilidade e suas articulações - se justifica pelas relações existentes entre tais áreas da matemática, que lidam com a incerteza em eventos aleatórios (em oposição a uma visão determinística da matemática, por vezes dominante quando se fala de uma ciência exata) e, desta forma, tratam do levantamento e análise de possibilidades. Os raciocínios relacionados a essas áreas constituem modos de pensamento lógico-matemático, visto que permitem que situações reais sejam exploradas e provêm formas de relacionar conjuntos de elementos. O trabalho visando o desenvolvimento desses raciocínios, no entanto, parece ganhar pouco espaço tanto na escolarização de jovens e adultos, quanto na de crianças e adolescentes, visto que estudos exploratórios realizados com diferentes modalidades e níveis de ensino apontam desempenhos insatisfatórios por parte dos estudantes (Bryant \& Nunes, 2012; Lima, 2010; Navarro-Pelayo, Batanero \& Godino, 1996, Pessoa, 2009, Silva, 2016, Souza \& Souza, 2019).

Na próxima seção são explorados os principais aportes teóricos adotados: a) a Teoria dos Campos Conceituais (Vergnaud, 1986, 1996), que ressalta, dentre outros aspectos, a 
importância do trabalho com diferentes situações que atribuem sentido aos conceitos para seu amplo entendimento; b) a organização de situações combinatórias proposta por Borba (2010), que as categoriza em problemas de arranjo, combinação, permutação e produto cartesiano; e c) a argumentação de Bryant e Nunes (2012), que apontam quatro requisitos ao amplo entendimento da probabilidade, por eles denominados demandas cognitivas, das quais, no presente texto, apresenta-se um recorte relacionado a uma delas: a construção de espaços amostrais.

\section{Aportes teóricos}

Um campo conceitual é definido como "um conjunto de situações, cujo domínio requer uma variedade de conceitos, de procedimentos e de representações simbólicas em estreita conexão" (Vergnaud, 1986, p. 10). Para esse autor um conceito é formado por um tripé de conjuntos: o conjunto das situações que atribuem sentido aos conceitos (S), o conjunto das propriedades e características imutáveis dessas diversas situações, isto é, seus invariantes (I) e o conjunto das representações simbólicas utilizadas para representá-las e que auxiliam na resolução de problemas (R). Vergnaud (1996) defende que "é através das situações e dos problemas a resolver que um conceito adquire sentido" (p. 156). Tal fala, e pressupostos acima citados, evidenciam o papel central do trabalho com uma pluralidade de situações na ampla compreensão de um conceito (e de conceitos a eles relacionados). Assim, no processo de ensino, conhecer e explorar problemas de naturezas variadas em sala de aula é de suma importância quando se visa o desenvolvimento dos conhecimentos dos estudantes.

Conceitos referentes à combinatória e à probabilidade estão inseridos no campo conceitual das estruturas multiplicativas, que, conforme apontado por Vergnaud (1996), diz respeito a "situações que exigem uma multiplicação, uma divisão ou uma combinação destas duas operações" (p. 167). No campo multiplicativo estão inclusos conceitos diversos, tais como a proporção, função linear, razão, produto cartesiano e outros conceitos combinatórios.

Batanero, Godino e Navarro-Pelayo (1996) apontam que a combinatória estuda os conjuntos discretos e as configurações que podem ser obtidas a partir de certas transformações que originam mudanças na estrutura da composição dos elementos desses conjuntos. Assim, é a área da Matemática que, entre outras coisas, "nos ensina a enumerar todos os modos possíveis que um dado número de objetos pode ser misturado e combinado de forma que tenhamos certeza de que nenhuma das possibilidades foi omitida" (p. 17). 
Reforçando a importância do trabalho com problemas combinatórios variados, Morgado, Pitombeira de Carvalho, Pinto de Carvalho e Fernandez (1991) apontam que a correta solução de um problema combinatório exige a plena compreensão da situação descrita no mesmo. Nesse sentido, adotou-se a proposta de Borba (2010), que integrando classificações anteriores e tomando por base invariantes de ordem e de escolha, distingue os problemas combinatórios em: arranjo, combinação, permutação e produto cartesiano. É válido destacar que tal autora considera, ainda, um terceiro invariante dos problemas combinatórios - o invariante de esgotamento, comum a todos os problemas citados, ou seja, a enumeração, direta ou indireta, de todas as possibilidades solicitadas no problema combinatório tratado.

Em problemas que exploram as situações de arranjo, combinação e permutação, as escolhas que constituem as diferentes possibilidades acontecem dentro de um único conjunto, podendo ser selecionados todos os elementos (caso dos problemas de permutação) ou alguns deles (em problemas de arranjo e de combinação). Dentre os quatro tipos de problemas combinatórios, apenas nos problemas de produto cartesiano a escolha se dá a partir de dois ou mais conjuntos. No que diz respeito à ordenação dos elementos na construção de possibilidades, este é um fator que determina possibilidades distintas nos problemas de arranjo e de permutação, mas não determina novas possibilidades nos problemas de combinação nem nos de produto cartesiano. Esses distintos tipos de situações combinatórias, dados os invariantes que as distinguem e diferentes representações simbólicas utilizadas na resolução de problemas que as exploram, permeiam as análises apresentadas mais adiante.

Por sua vez, a probabilidade é definida por Morgado et al. (1991) como "o ramo da Matemática que cria, desenvolve e, em geral, pesquisa modelos que podem ser utilizados para estudar experimentos ou fenômenos aleatórios” (p. 119). A probabilidade trata, dessa forma, do estudo de eventos que ocorrem aleatoriamente, isto é, que não são determinísticos: "as pessoas sabem que eles podem acontecer, mas não têm certeza se e quando eles acontecerão" (Bryant \& Nunes, 2012, p. 3).

Bryant e Nunes (2012) argumentam que a probabilidade é um conceito complexo e apontam quatro demandas cognitivas ao seu amplo entendimento e, logo, ao desenvolvimento do raciocínio probabilístico. Estas demandas são: entendimento da aleatoriedade, construção e análise de espaço amostral, comparação e quantificação de probabilidades, e compreensão de correlações (relações entre eventos). Neste texto, tem-se por foco o trabalho com o espaço 
amostral. Para esses autores, levantar e entender espaços amostrais é um passo importante não só para o cálculo de probabilidades, mas um elemento essencial à compreensão da natureza do conceito de probabilidade. Eles afirmam que "problemas em probabilidade são sempre sobre uma gama de eventos possíveis, mas incertos, que ocorrem aleatoriamente, [...] nos quais nós temos que saber precisamente quais são todos os eventos possíveis” (p. 29).

A construção do conjunto composto pelas diferentes possibilidades em uma situação aleatória (espaço amostral) está intrinsecamente relacionada ao raciocínio combinatório, visto que o levantamento de tais possibilidades depende da aplicação de conhecimentos e estratégias tipicamente combinatórias. Na outra direção, a explicitação de todas as possibilidades (quando viável) ao se resolver um problema combinatório relaciona-se ao conceito probabilístico de espaço amostral e, a partir do uso de estratégias diversas e suas respectivas representações, consiste em uma oportunidade de reflexão dos invariantes da situação combinatória envolvida no problema, visto que é possível analisar diferentes possibilidades uma a uma. Essa relação mais direta entre combinatória e probabilidade (diferentes situações combinatórias e espaços amostrais) é o foco do presente artigo, cujo método é descrito a seguir.

\section{Procedimentos metodológicos}

A pesquisa foi realizada junto a 24 estudantes cursando diferentes etapas da Educação de Jovens e Adultos, em escolas públicas localizadas em Pernambuco, no nordeste do Brasil. Estes estudantes são referidos no presente texto como P1, P2, P3 .., P24 e pertenciam a três grupos de 8 estudantes cada, cursando etapas equivalentes à conclusão dos anos iniciais do ensino fundamental, dos anos finais ou do ensino médio. Os estudantes do Grupo 1 (concluintes dos anos iniciais) apresentaram média de 42 anos, os do Grupo 2 (concluintes dos anos finais) média de 32 anos e os do Grupo 3 (concluintes do ensino médio) de 33 anos, quase idêntica a do grupo anterior.

Foram realizadas entrevistas clínicas individuais, utilizando-se o método piagetiano, que permite ao pesquisador "compreender como o sujeito pensa, como analisa situações, como resolve problemas, como responde às contra sugestões do examinador" (Carraher, 1998, p. 6). Tal método de coleta de dados foi escolhido visando potencializar a interação entre pesquisadora e estudante(s), no sentido de investigar de maneira mais aprofundada as escolhas e ações feitas frente à resolução dos 20 problemas propostos: quatro combinatórios (produto 
cartesiano, combinação, permutação e arranjo) e 16 probabilísticos (organizados em quatro blocos contendo quatro problemas cada e relacionados a cada um dos problemas combinatórios citados - referentes a diferentes demandas cognitivas).

No que diz respeito à articulação entre os problemas combinatórios e probabilísticos, esta se deu a partir de revisitações propostas em dois tipos de testes. Metade dos participantes de cada grupo resolveu o Teste 1, no qual foram apresentados cada problema combinatório seguido pelo bloco de problemas probabilísticos a ele relacionado. Por sua vez, os demais participantes de cada grupo resolveram o Teste 2, sendo os blocos de problemas probabilísticos resolvidos antes dos problemas combinatórios a eles correspondentes.

No presente texto, tem-se por foco, especificamente, a articulação entre os problemas de produto cartesiano, combinação, permutação e arranjo (Figura 1) e os de construção de espaço amostral a eles correspondentes (Figura 2). Resultados preliminares nesse sentido, referentes a um estudo piloto da pesquisa, foram publicados em Lima e Borba (2017). Neste tipo de problema probabilístico, que explora uma das demandas cognitivas ao amplo entendimento da probabilidade, foi solicitada aos estudantes a construção de uma listagem contendo todas as possibilidades referentes a cada situação combinatória. É importante destacar que a listagem é uma representação que surge espontaneamente ao se resolver problemas de contagem. Contudo, a partir da proposta de articulação via construção de espaços amostrais, demandando diretamente o uso de tal estratégia, teve-se o intuito de garantir que todos os participantes a utilizariam em algum momento (fosse na revisitação ao problema combinatório - Teste 1 - ou no primeiro contato com o mesmo - Teste 2) para indicar as diversas possibilidades em cada situação combinatória.

As análises quantitativas dos desempenhos apresentados pelos participantes foram realizadas com auxílio do software Statistical Package for the Social Sciences (SPSS), permitindo que fossem feitas análises descritivas de desempenho considerando-se tipo de problema, representação e estratégia utilizada pelos participantes, bem como a influência da ordem de apresentação dos problemas (tipo de teste) e do nível de escolarização nos desempenhos apresentados.

No que diz respeito à atribuição de pontuação aos desempenhos apresentados pelos estudantes quando da resolução dos problemas combinatórios e de construção de espaço amostral propostos, atribuiu-se zero (0) pontos quando evidenciou-se (seja por meio da 
quantificação ou da enumeração das mesmas) menos da metade do número total de possibilidades, um (1) ponto quando evidenciou-se metade ou mais das possibilidades e dois (2) pontos quando houve esgotamento de possibilidades, isto é, um acerto total. Na seção a seguir são apresentados e discutidos os resultados obtidos.

Figura 1. Problemas combinatórios propostos
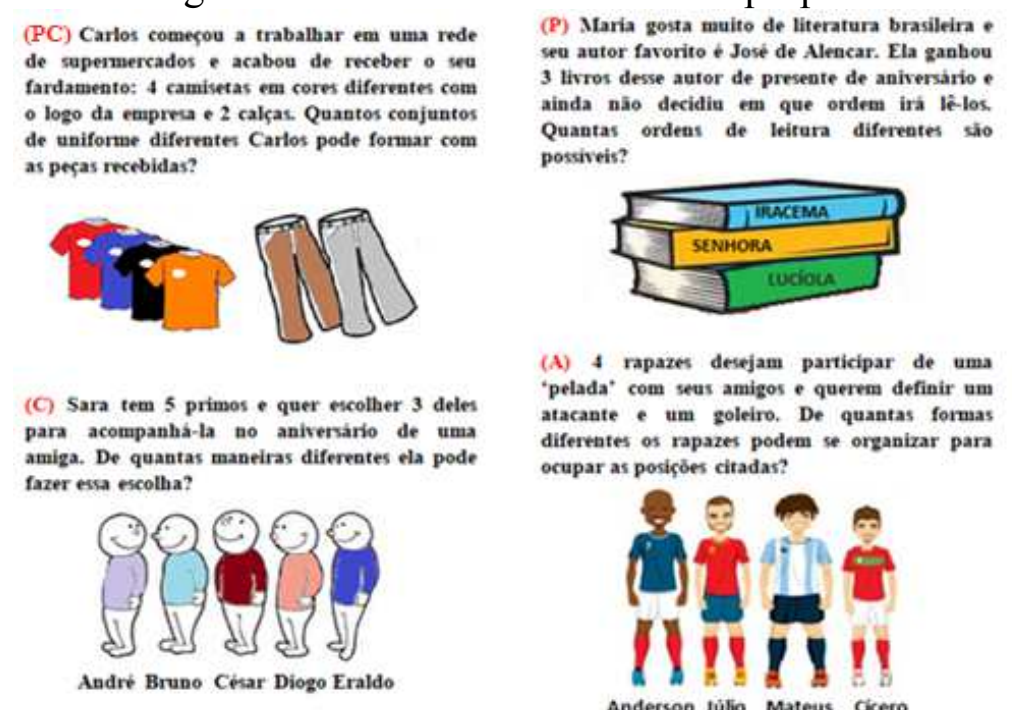

(A) 4 rapazes desejam participar de uma 'pelada' com seus amigos e querem definir um atacante e um goleiro. De quantas formas diferentes os rapazes podem se organizar para ocupar as posiçōes citadas?

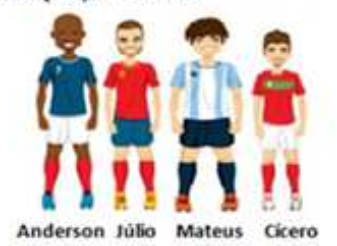

PC: produto cartesiano: 8 possibilidades; C: combinação: 10 possibilidades; P: permutação: 6 possibilidades; A: arranjo: 12 possibilidades.

\section{Fonte: Lima (2018, p. 69)}

Figura 2. Problemas probabilísticos propostos (construção de espaços amostrais)

(EAPC) Liste todos os conjuntos de calça e camiseta que Carlos pode formar com as peças de roupas recebidas.

(EAC) Liste todas as formas como Sara pode escolher $\mathbf{3}$ dos seus 5 primos para acompanhà-la à festa.

(EAP) Liste todas as ordens de leitura que Maria pode escolher com os 3 livros que ganhou de presente.

(EAA) Liste todas as formas pelas quais se pode escolher 2 rapazes para ocupar as posições de goleiro e de atacante a partir dos 4 amigos.

EAPC: construção de espaço amostral de produto cartesiano;

EAC: construção de espaço amostral de combinação;

EAP: construção de espaço amostral de permutação;

EAA: construção de espaço amostral de arranjo.

Fonte: As autoras

\section{Apresentação e discussão dos resultados}

A seguir são apresentados e discutidos os resultados referentes: a) ao desempenho médio (geral e por grupo de participantes) nos diferentes tipos de problemas propostos; b) representações e estratégias utilizadas pelos participantes; c) as revisitações propostas nos dois tipos de teste (combinatória $\rightarrow$ probabilidade; probabilidade $\rightarrow$ combinatória). 


\section{Desempenho por tipo de problema}

De acordo com as pontuações atribuídas aos desempenhos dos participantes da pesquisa, era possível que estes obtivessem um desempenho de 0,1 ou 2 pontos em cada problema combinatório ou probabilístico (construção de espaço amostral) resolvido. $\mathrm{Na}$ Tabela 1 são apresentados os resultados gerais referentes aos quatro problemas combinatórios propostos, bem como no que diz respeito aos problemas de construção de espaço amostral a eles correspondentes.

O maior desempenho médio observado, tanto nos problemas combinatórios quanto nos de construção de espaço amostral, refere-se ao problema de produto cartesiano. Por sua vez, os menores desempenhos médios foram observados no que diz respeito ao problema de combinação (combinatório e espaço amostral), dado que reflete dificuldades apresentadas pelos participantes quanto à compreensão dos invariantes desse tipo de situação combinatória. Por sua vez, os desempenhos médios nos problemas combinatórios de permutação e de arranjo foram idênticos e bem próximos no que diz respeito à explicitação de espaço amostral correspondente. Nesses dois tipos de problemas a ordenação dos elementos determina possibilidades distintas. Incompreensões nesse sentido, erros de ordem, são ressaltadas por Navarro-Pelayo, Batanero e Godino (1996) e podem justificar os desempenhos (baixos e semelhantes) observados. Pesquisas tais como a de Fernandes, Correia e Guzmán (2010) e a de Zwanch (2019) enfocam situações combinatórias (inclusive articuladas à probabilidade) nas quais os estudantes apresentam dificuldades pelo mesmo motivo.

Tabela 1.

Desempenho médio nos problemas combinatórios e de construção de espaços amostrais (pontuação máxima de 2 pontos por problema)

Problema combinatório

\begin{tabular}{llr}
\hline Produto cartesiano & 1,17 & 1,38
\end{tabular}

Construção de Espaço Amostral

0,25

\section{Permutação}

0,75

0,75

0,71

Fonte: As autoras 
Tais resultados corroboram achados de estudos anteriores, como os de Azevedo (2013), Lima (2010) e Pessoa (2009), que apontam os problemas que abordam a situação de produto cartesiano como os de mais simples resolução dentre os combinatórios. Atribui-se essa maior facilidade dos estudantes (de diferentes níveis e modalidades de ensino) com essa situação combinatória em específico ao fato de que esse tipo de problema combinatório é, geralmente, o mais trabalhado desde o início da escolarização - inclusive por sua resolução estar diretamente relacionada à operação de multiplicação. Analogamente, tais estudos anteriores (Azevedo, 2013; Lima, 2010; Pessoa, 2009) apontam um desempenho insatisfatório em problemas de combinação. $\mathrm{O}$ fato de a ordem não determinar novas possibilidades nesse tipo de situação combinatória levou alguns dos participantes do estudo a terem dificuldades em esgotar o número de possibilidades, bem como, por vezes, extrapolaram tal número (por considerar erroneamente o invariante de ordem, apontando casos repetidos) - seja por estratégias diversas ou pela listagem solicitada no problema de espaço amostral correspondente.

Outro dado importante da pesquisa, que evidencia a maior dificuldade no problema de combinação, diz respeito aos percentuais de acertos totais observados. Assim, destaca-se que, os casos nos quais houve esgotamento de possibilidades nos problemas combinatórios, os percentuais foram iguais a: 29,2\% no problema de produto cartesiano, 20,8\% no problema de arranjo, $12,5 \%$ no problema de permutação e apenas $8,3 \%$ no problema de combinação.

Tais dados corroboram os desempenhos observados nos diferentes problemas combinatórios, bem como nos problemas de construção de espaço amostral a eles correspondentes (Tabela 1), reforçando que os participantes da presente pesquisa apresentaram maior facilidade ao levantar possibilidades referentes à situação de produto de medidas e maior dificuldade relacionada à situação de combinação. Por sua vez, apresentaram dificuldades intermediárias e desempenhos semelhantes entre si no que diz respeito às situações de arranjo e de permutação, nas quais a compreensão dos invariantes de ordem assume grande importância.

Além de voltar o olhar ao tipo de problema (combinatório ou probabilístico correspondente - construção de espaço amostral), outra importante variável considerada nas análises está relacionada à influência do nível de escolarização dos participantes nos desempenhos apresentados pelos três grupos: Grupo 1: Estudantes concluintes dos anos 
iniciais do ensino fundamental; Grupo 2: Estudantes concluintes dos anos finais do ensino fundamental e Grupo 3: Estudantes concluintes do ensino médio. Tais resultados são apresentados na Figura 3 (combinatórios) e na Figura 4 (construção de espaços amostrais).

Figura 3. Desempenho médio nos problemas combinatórios (por grupo); (pontuação máxima de 2 pontos por problema)

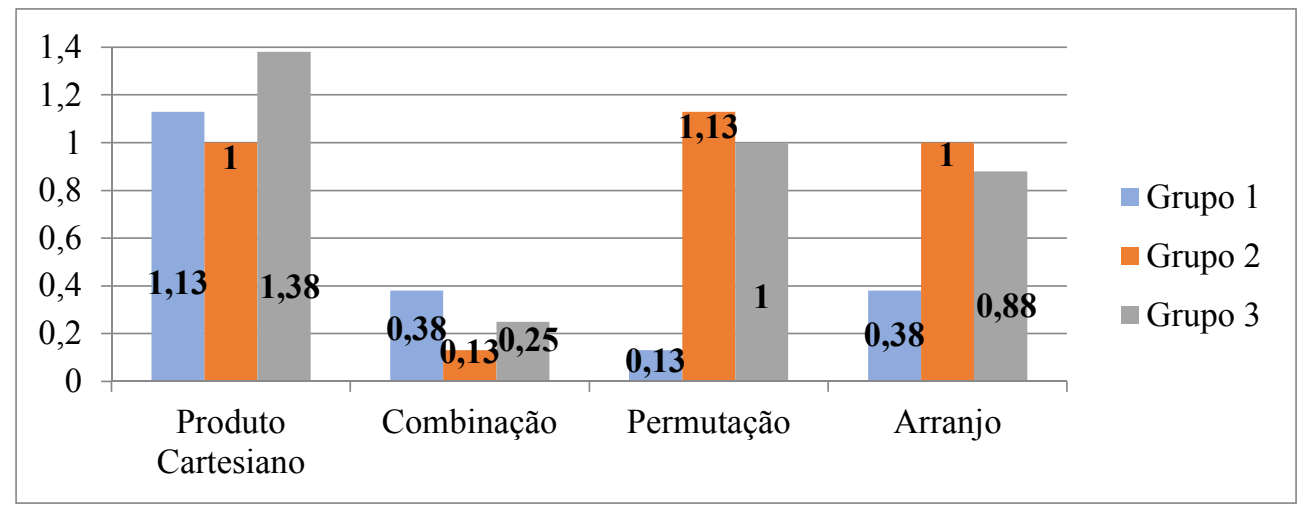

Fonte: Lima (2018, p. 84).

Os resultados ilustrados na Figura 3 mostram que a escolarização não teve, de modo geral, uma influência direta expressiva nos desempenhos apresentados. No que diz respeito aos problemas de produto de medidas e de combinação (nos quais o melhor e o pior desempenho médio foram observados, respectivamente), o desempenho foi semelhante nos três grupos de estudantes. Por sua vez, nos problemas que exploram as situações combinatórias de arranjo e de permutação foi possível perceber que estudantes dos Grupos 2 e 3 tenderam a obter desempenhos superiores aos de estudantes do Grupo 1, mas próximos entre si. Nesse grupo (Grupo 1) as incompreensões referentes à ordenação em tais problemas foram mais expressivas - o que levou ao desempenho inferior em relação aos demais grupos de participantes.

Reforçando o observado anteriormente (Figura 3), a Figura 4 aponta que, em geral, houve pequenas melhorias quantitativas nos desempenhos médios dos participantes da pesquisa com o avanço da escolarização ao construir espaços amostrais articulados a situações combinatórias, ou seja, a partir da solicitação de que os participantes apresentassem uma listagem/indicação uma a uma das possibilidades consideradas. É importante ressaltar que o desempenho dos estudantes do Grupo 3 avançou bastante no problema de produto cartesiano, 
problema no qual, como evidenciado em análises a seguir, participantes de diferentes grupos avançaram qualitativamente. Para o Grupo 3, no entanto, a chegada ao esgotamento de possibilidades foi mais frequente.

Figura 4. Desempenho médio nos problemas de construção de espaço amostral (por grupo); (pontuação máxima de 2 pontos por problema)

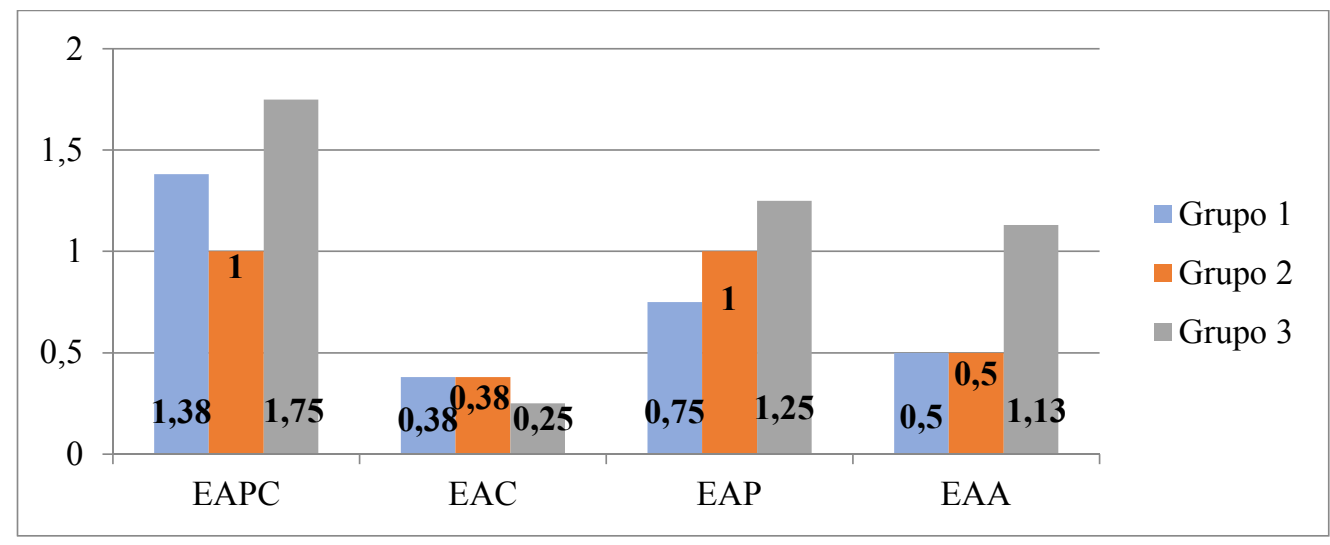

EAPC: espaço amostral de produto de medidas; EAC: espaço amostral de combinação; EAP: espaço amostral de permutação; EAA: espaço amostral de arranjo.

Fonte: Lima (2018, p. 87).

Dado o posto até então, mesmo que os desempenhos referentes aos diferentes tipos de situações combinatórias (e também à construção de seus respectivos espaços amostrais) tenham sido diferentes entre si, as dificuldades enfrentadas pelos participantes da pesquisa com diferentes níveis de escolarização foram semelhantes. Esses resultados evidenciam o papel central das situações, bem como a compreensão de seus invariantes (propriedades e relações constantes) nos desempenhos apresentados e corroboram a defesa de que diferentes tipos de problemas combinatórios sejam explorados em sala de aula ao longo da educação básica para que o raciocínio combinatório dos estudantes seja amplamente desenvolvido (Borba, 2010). Por outro lado, qualitativamente, ganha espaço, ainda, o terceiro componente do tripé apontado por Vergnaud $(1986,1996)$ : as representações simbólicas. Análises nesse sentido são apresentadas na subseção que segue.

\section{Um olhar para as representações simbólicas e estratégias}

$\mathrm{Na}$ Tabela 2 são apresentadas as diferentes estratégias e respectivas representações utilizadas pelos estudantes ao resolverem os problema combinatórios do Teste 1 (no qual tais problemas foram resolvidos antes de serem revisitados via construção de espaços amostrais) e 
do Teste 2 (no qual a listagem havia sido solicitada previamente, consistindo, logo, em uma revisitação ao problema probabilístico correspondente - espaço amostral).

Tabela 2.

Percentuais de estratégias e representações utilizadas nos problemas combinatórios em ambos os tipos de teste (percentual)

$\begin{array}{cccc}\text { Produto } & \text { Combinação } & \text { Permutação } & \text { Arranjo } \\ \text { Cartesiano } & & \end{array}$

Teste 1 Teste 2 Teste 1 Teste 2 Teste 1 Teste 2 Teste 1 Teste 2

Sem revisitação

Enumeração oral

Listagem extensiva

Listagem reduzida

Generalização de listagem

Uso de valor do enunciado

Adição

Multiplicação inadequada
25

75
58,3

25

8,3

8,3

41,6

66,8

8,3

8,3

8,3

$8,3 \quad 8,3$

$8,3 \quad 16,7$

58,4

$33,5 \quad 83,4$

25

$8,3 \quad 8,3$

8,3

$8,3 \quad 8,3$

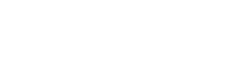

$16,7 \quad 16,7 \quad 8$

8,3

$8,3 \quad 8,3$

Multiplicação adequada $\quad 16,7 \quad 16,7$

Fonte: As autoras

As categorias referentes às estratégias e respectivas representações apresentadas na Tabela 2 foram elencadas à posteriori, isto é, a partir da análise das resoluções dos problemas propostos por participantes da pesquisa. O uso de enumeração oral, estratégia mais utilizada no que se refere à resolução de problemas combinatórios no Teste 1, bem como muito presente nas revisitações do Teste 2 , diz respeito a situações nas quais os estudantes indicaram oralmente diferentes possibilidades, sem fazer registro de cada uma delas. Nesse caso, os registros escritos dos participantes que fizeram uso de tal estratégia e respectiva representação nas folhas de teste disponibilizadas continham meramente a resposta final obtida (o número de possibilidades considerado).

Por sua vez, o uso (espontâneo) de listagem para resolver problemas combinatórios, no Teste 1, consistiu na indicação, por escrito, das possibilidades levantadas. Essa estratégia, com 
representação escrita, foi mais utilizada por estudantes dos Grupos 2 e 3, os quais possuíam melhor domínio da escrita do que os estudantes do Grupo 1. Por vezes, tais listagens foram compostas pela escrita completa dos nomes de elementos/cores/pessoas presentes nos enunciados dos problemas propostos (listagem extensiva) e, em outros casos, os estudantes fizeram uso de abreviações/iniciais (listagem reduzida - exemplificada na resolução do estudante P17 (Grupo 3). Tal estudante utilizou as iniciais dos livros envolvidos no problema de permutação (I, S e L) ao apresentar cinco das seis possibilidades existentes no contexto do problema cujo enunciado era: Maria gosta muito de literatura brasileira e seu autor favorito é José de Alencar. Ela ganhou 3 livros desse autor de presente de aniversário e ainda não decidiu em que ordem irá lê-los. Quantas ordens de leitura diferentes são possíveis?.O estudante escreveu, então, o quantitativo encontrado seguido da listagem reduzida das possibilidades consideradas: 5, ISL, LSI, ILS, LIS, SLI..

Em alguns casos, os estudantes utilizaram, ainda, cálculos numéricos para determinar o número de possibilidades em cada problema combinatório em questão - adição e multiplicação (operação adequada no caso de produto cartesiano, porém não nos demais) - ou usaram valores presentes nos enunciados dos problemas para dar suas respostas. Esta última estratégia, em especial, evidencia incompreensões do invariante de escolha dos problemas combinatórios que envolvem as diferentes situações, pois tal escolha é essencial para construção de possibilidades em cada caso. É válido destacar que o uso incorreto dos valores presentes nos enunciados foi utilizado exclusivamente por estudantes do Grupo 1 (concluintes dos anos iniciais), como exemplificado nas transcrições apresentadas na Figura 5.

Tais transcrições evidenciam incompreensões de participantes do Grupo 1 referentes aos invariantes de escolha dos problemas combinatórios (produto cartesiano e permutação). Nos dois casos, os próprios elementos são incorretamente interpretados como possibilidades. No problema de produto cartesiano, P3 somou os elementos dos dois conjuntos de peças de roupa (camisetas e calças), chegando ao total de seis elementos. Em sua fala, deixou claro considerar esse valor o número de conjuntos de uniformes distintos que podem ser formados no contexto do problema em questão. Por sua vez, ao resolver o problema de permutação, P5 usou o valor presente no enunciado do problema (número de livros) e o apontou como sendo o número total de possibilidades na situação (ordens de leitura). É importante destacar, ainda, a dificuldade com a escrita apresentada pela grande maioria dos participantes do Grupo 1 (o que 
inviabilizou o registro das estratégias e representações utilizadas) que ressalta a importância da condução de entrevistas clínicas, pois em suas falas foi possível compreender como estes estudantes pensaram para resolver cada problema.

Figura 5. Exemplos de transcrições das entrevistas clínicas (Grupo 1)

Transcrição 1. Problema de produto de medidas (Teste 1), resolvido por P3 (Grupo 1). Adição: nenhuma possibilidade levantada

Carlos começou a trabalhar em uma rede de supermercados e acabou de receber o seu fardamento: 4 camisetas em cores diferentes com o logo da empresa e 2 calças. Quantos conjuntos de uniforme diferentes Carlos pode formar com as peças recebidas?

Pesquisadora: "O senhor escreveu que Carlos pode formar seis conjuntos de uniforme diferentes. Como o senhor resolveu?"

P3: “[...] ele recebeu quatro camisetas e duas calças, "né'? [...] ele pode formar seis conjuntos."

Pesquisadora: "Por quê?"

P3: "Cada camiseta dessa é uma farda [...] e cada calça é uma farda, 'né'? Eu pensei assim: quatro e dois dá seis."

Transcrição 2. Problema de permutação (Teste 1), resolvido por P5 (Grupo 1). Uso de valor do enunciado: nenhuma possibilidade levantada

Maria gosta muito de literatura brasileira e seu autor favorito é José de Alencar. Ela ganhou 3 livros desse autor de presente de aniversário e ainda não decidiu em que ordem irá lê-los. Quantas ordens de leitura diferentes são possíveis?

P5: "Eu acho que é de três formas, por que ela ganhou três livros, aí cada livro é uma forma, "né'?" Pesquisadora:"[...] Como o senhor leria? Em qual ordem?" [...]

Pesquisadora: "O senhor me falou Iracema, Senhora e Lucíola, essa é uma forma?" P5: "Três formas."

\section{Fonte: As autoras}

As discussões anteriores e a sistematização apresentada adiante na Tabela 3, referentes ao uso de estratégias com representações simbólicas mais refinadas e/ou adequadas em função do grupo de participantes, lançam destaque à importância da escolarização na construção de conhecimentos acerca da combinatória, bem como de tais representações.

Os estudantes dos grupos 2 e 3 fizeram uso, com maior frequência, de estratégias e representações simbólicas mais eficientes à resolução dos problemas propostos (listagens reduzidas e/ou sistemáticas - e multiplicação), mas é válido ressaltar que estas podem ainda ser insuficientes quando se lida com problemas mais complexos ou que envolvem um maior número de possibilidades. A escolarização formal possui, assim, papel central na construção (contínua e ao longo da escolarização) de um repertório de estratégias e suas representações 
relacionadas ao raciocínio combinatório, chegando-se, inclusive, ao uso de fórmulas (Borba, 2016).

Tabela 3.

Percentuais de estratégias e representações simbólicas mais utilizadas (por grupo)

\begin{tabular}{|c|c|c|c|}
\hline & $\begin{array}{c}\text { Grupo 1. Concluintes dos } \\
\text { Anos Iniciais }\end{array}$ & $\begin{array}{c}\text { Grupo 2. Concluintes dos } \\
\text { Anos Finais }\end{array}$ & $\begin{array}{c}\text { Grupo 3. Concluintes do } \\
\text { Ensino Médio }\end{array}$ \\
\hline $\begin{array}{l}\text { Problemas } \\
\text { combinatórios }\end{array}$ & $\begin{array}{l}\text { - Sem revisitação }(37,5 \%) ; \\
\text { - Enumeração Oral }(34 \%) ; \\
\text { - Adição ou uso de valores do } \\
\text { enunciado }(16 \%) \text {. }\end{array}$ & $\begin{array}{l}\text { - Enumeração Oral }(78 \%) \\
\text { - Sem revisitação }(16 \%) \\
\text { - Listagens escritas }(4 \%)\end{array}$ & $\begin{array}{l}\text { - Enumeração Oral (47\%); } \\
\text { - Listagens escritas (16\%); } \\
\text { - Multiplicação }(22 \%) \text {. }\end{array}$ \\
\hline $\begin{array}{l}\text { Espaços } \\
\text { amostrais }\end{array}$ & $\begin{array}{l}\text { - Enumeração Oral (72\%); } \\
\text { - Listagem Extensiva (28\%). }\end{array}$ & $\begin{array}{l}\text { - Listagem extensiva } \\
\text { (algumas sistemáticas) } \\
(84 \%) .\end{array}$ & $\begin{array}{l}\text { - Listagem não sistemática } \\
\text { (reduzidas ou extensivas) } \\
(75 \%) \text {; } \\
\text { - Sistematização e } \\
\text { generalização }(25 \%) \text {. }\end{array}$ \\
\hline
\end{tabular}

Fonte: As autoras

No caso específico da construção de espaços amostrais a dificuldade com a escrita e a própria familiarização com problemas combinatórios e matemáticos em geral, foram determinantes no uso de diferentes estratégias e representações para levantar possibilidades uma a uma (seja a partir da enumeração oral ou de listagens de diferentes naturezas e níveis de apropriação/generalização), como se pode observar na Tabela 4.

Tabela 4.

Representações simbólicas/estratégias utilizadas nos problemas de construção de espaços amostrais (percentual)

\begin{tabular}{lcccc}
\hline Estratégias/ & \multicolumn{3}{c}{ Espaço amostral } \\
Representações & 20,8 & 25 & 25 & 25 \\
\cline { 2 - 5 } & $\begin{array}{c}\text { Produto } \\
\text { cartesiano }\end{array}$ & Combinação & Permutação & Arranjo \\
\hline Enumeração oral & 58,3 & 66,6 & 62,5 & 41,6 \\
Listagem extensiva não sistemática & 16,7 & & & 16,7 \\
Listagem extensiva sistemática & & 4,2 & 8,3 & 4,2 \\
Listagem reduzida não sistemática & & & 4,2 & 8,3 \\
Listagem reduzida sistemática & 4,2 & 4,2 & & 4,2 \\
\hline Generalização de listagem & & & &
\end{tabular}

Fonte: As autoras

Os resultados obtidos a partir da pesquisa desenvolvida evidenciaram que a 
construção de espaços amostrais (a partir da solicitação de listagem/indicação de cada possibilidade - enumeração oral no caso dos estudantes com dificuldades de escrita) foi um caminho rico para a discussão e reflexão acerca dos invariantes (relações de ordem e de escolha) dos distintos contextos combinatórios explorados.

\section{O papel das revisitações}

Ao terem um contato inicial com as situações combinatórias de produto cartesiano, combinação, permutação e arranjo a partir da solicitação de construção de espaço amostral (caso de Teste 2) ou a revisitar tais situações via raciocínio probabilístico após resolvê-las inicialmente fazendo uso de estratégias e representações livres (Teste 1) os estudantes tiveram a chance de analisar, de maneira mais sistematizada, as possibilidades já consideradas e levantar outras à luz dos invariantes de ordem e de escolha considerados em cada situação. A partir dessa articulação entre combinatória e probabilidade os desempenhos dos diferentes grupos tenderam a se aproximar.

O desempenho dos participantes do Grupo 3 (concluintes do Ensino Médio) se destacou no problema de espaço amostral de produto cartesiano, visto que os estudantes de tal grupo conseguiram esgotar, na maior parte das vezes, as possibilidades relacionadas a esses problema em específico, problema no qual o melhor desempenho geral foi observado - sendo assim, o tipo de problema mais simples para os participantes do estudo como um todo.

Figura 6.

Desempenho médio nos problemas combinatórios e de espaço amostral (por tipo de teste)

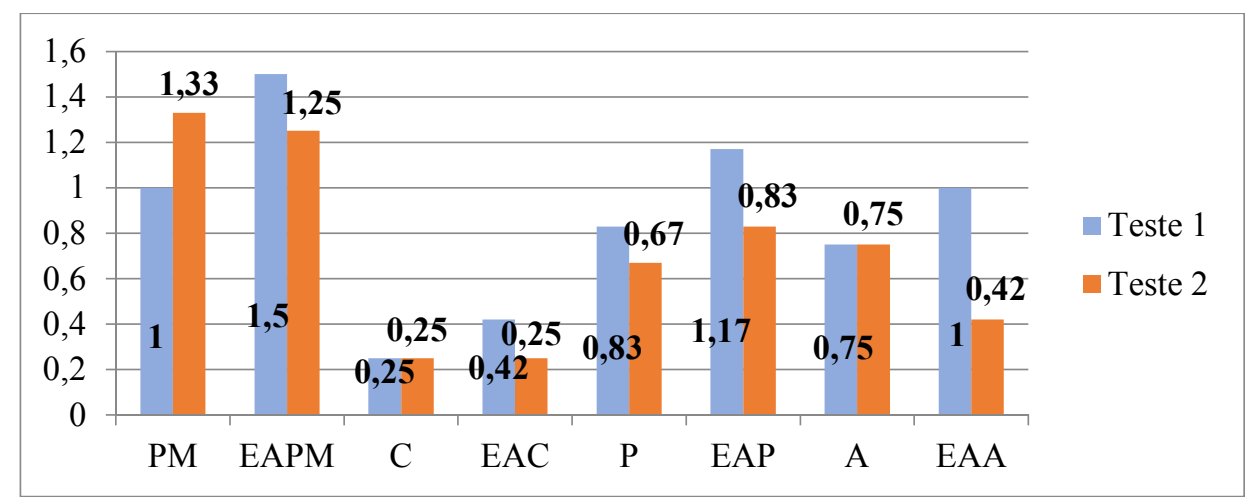

PC: produto cartesiano; EAPC: espaço amostral de produto cartesiano; C: combinação; EAC: espaço amostral de combinação; P: permutação; EAP: espaço amostral de permutação; A: arranjo; EAA: espaço amostral de arranjo; Teste 1: Problemas combinatórios revisitados via probabilidade; Teste 2: Problemas probabilísticos revisitados via combinatória.

Fonte: Lima (2018, p. 89) 
O gráfico apresentado na Figura 6 evidencia diferentes comportamentos referentes aos desempenhos apresentados pelos participantes da pesquisa a depender do tipo de teste resolvido isto é, da ordem de apresentação dos problemas propostos. Nos casos nos quais os problemas combinatórios foram resolvidos inicialmente (Teste 1) e revisitados via construção de espaço amostral o desempenho médio tendeu a ser melhor nesses problemas de natureza probabilística nos quais foi solicitada a indicação das possibilidades uma a uma. Essa revisitação via espaço amostral proporcionou um momento de (re)avaliação das resoluções dadas aos problemas combinatórios no Teste 1 , permitindo que os participantes realizassem correções e até mesmo mudassem as estratégias e representações utilizadas na busca da explicitação de mais alguma possibilidade (ou até mesmo o esgotamento: indicação de todas as possibilidades).

Por sua vez, ao se tratar do Teste 2, no qual os problemas de construção de espaços amostrais eram resolvidos antes dos problemas combinatórios sem solicitação específica de listagem/enumeração de possibilidades, houve um pequeno crescimento no desempenho médio apenas nos problemas de produto cartesiano e de arranjo. Dessa maneira, as revisitações propostas no Teste 2 (problema combinatório como revisitação ao problema de explicitação de espaço amostral) não surtiram o mesmo efeito daquelas presentes no Teste 1, não proporcionando um avanço, que é em sua maioria qualitativo, como mencionado no caso anterior.

Os avanços qualitativos que são frutos dessa articulação entre combinatória e probabilidade (mais presentes no Teste 1), no entanto, não são claramente evidenciados em análises como as até então apresentadas. As pontuações atribuídas aos desempenhos apresentados se basearam em categorias de desempenho, assim, um estudante que tivesse indicado metade das possibilidades relativas a certo problema combinatório, precisaria conseguir, a partir da revisitação do mesmo, esgotá-las para que pudesse avançar de categoria (aumentar sua pontuação). Isso justifica o avanço quantitativo expressivo observado exclusivamente ao se tratar do problema de produto cartesiano no Teste 1, pois, além de essa ter sido a situação combinatória mais simples para os participantes do estudo, a revisitação por meio da listagem/enumeração oral de cada possibilidade (construção de espaço amostral) levou muitos estudantes a esgotarem as possibilidades (esgotamento que se mostrou mais difícil nas demais situações combinatórias propostas). 
Assim, é de suma importância destacar que os problemas propostos, que articularam diferentes situações combinatórias à construção de espaços amostrais proporcionaram melhorias de desempenho não só no sentido de explicitação de um número maior de possibilidades (por vezes se aproximando bastante de seu esgotamento), como também permitiram que os estudantes pudessem refletir acerca dos invariantes de ordem e de escolha das situações combinatórias em questão e modificar suas estratégias de resolução de tais problemas.

Os participantes que resolverem o Teste 1 (problemas combinatórios revisitados via construção de espaços amostrais) que não haviam utilizado a listagem/enumeração espontaneamente, tiveram a chance, na revisitação, de indicar uma a uma as possibilidades referentes a cada problema combinatório proposto. Por sua vez, os estudantes que já haviam utilizado algum tipo de listagem ao resolver os problemas combinatórios de produto de medidas, combinação, permutação e arranjo puderam, no momento das revisitações a partir do raciocínio probabilísticos, revisar e fazer ajustes em tais registros. Esse momento de revisitação/análises/ajustes permitiu que fossem levantadas reflexões sobre os invariantes das situações combinatórias em questão, na busca pelo esgotamento dos casos possíveis em cada contexto. A transcrição exemplificada na Figura 7 ilustra contribuições que a revisitação aos problemas combinatórios via construção de espaços amostrais proporcionou aos desempenhos dos participantes.

Figura 7. Exemplo de transcrição da entrevista clínica (P17)

Transcrição 3: Problema de arranjo (Teste 1), resolvido por P17 (Grupo 3). Enumeração oral: mais da metade das possibilidades

4 rapazes desejam participar de uma 'pelada' com seus amigos e querem definir um atacante e um goleiro. De quantas formas diferentes os rapazes podem se organizar para ocupar as posições citadas?

Pesquisadora: "[...] você já me falou Júlio e Cícero. Desses dois quem vai ser o goleiro e quem vai ser o atacante?"

P17: "O goleiro vai ser Júlio e o atacante vai ser Cícero..."

[...] P17 indica oralmente outra dupla de rapazes.

Pesquisadora:"Quantas formas são possíveis? Duas?"

P17: "São quatro. Pode ser o contrário também!"

Após passar a considerar a inversão de posições, registra as letras A (atacante) e G (goleiro) acima de cada desenho representando os rapazes. Identifica mais dois pares (e também seus inversos), chegando a enumerar oralmente oito possibilidades.

Fonte: As autoras 
Como pode ser observado, o participante P17 já havia demonstrado compreender os invariantes de ordem e de escolha referentes ao problema de arranjo proposto, no qual, inicialmente, utilizou a enumeração oral para indicar 8 possibilidades (Júlio-Cícero; CíceroJúlio; Anderson-Mateus; Mateus-Anderson; Anderson-Cícero; Cícero-Anderson; MateusJúlio; Júlio-Mateus). Ele, assim, havia escolhido corretamente dois dos rapazes por vez e percebido que a ordem indicaria possibilidades distintas, referentes a qual dos rapazes seria atacante e qual seria goleiro, mas não havia conseguido esgotar as possibilidades.

A partir da revisitação ao problema em questão, via construção do espaço amostral correspondente, o estudante fez uso de listagem reduzida sistemática (utilizando abreviações dos nomes de cada um dos rapazes mencionados no enunciado e 'At' para atacante e 'Go' para goleiro e conseguiu identificar novas possibilidades válidas no problema em questão. Dessa maneira, conseguiu atingir um acerto total, esgotando o número de possibilidades (12).

A construção do espaço amostral atuou, nesse caso, como um importante auxílio na chegada de um acerto total, pois facilitou a visualização das possibilidades consideradas pelo estudante e permitiu que ele sistematizasse a contagem dos pares - também invertidos. Dessa forma, a compreensão dos invariantes da situação combinatória resolvida foi potencializada pela revisitação sob o olhar da probabilidade.

Destaca-se que a chegada ao esgotamento de possibilidades não foi a única contribuição evidenciada a partir da revisitação aos problemas combinatórios. Em alguns casos, tais revisitações proporcionaram a reflexão sobre os invariantes dos problemas em questão que levaram os estudantes a desconsiderarem possibilidades repetidas ou indicarem mais algumas possibilidades, sem que se chegasse a um acerto total.

Ao resolver o problema combinatório referente à situação de permutação de três livros, o participante P1 (concluinte dos Anos Iniciais) fez uso de multiplicação inadequada. O estudante considerou o valor presente no enunciado (número de livros) e o multiplicou por ele mesmo - 3 vezes 3. A partir dessa operação, P1 indicou que existiam nove ordens de leitura distintas no contexto em questão, sem especificar (enumerar oralmente ou listar) nenhuma delas. Posteriormente, a partir da revisitação a tal situação combinatória via construção do espaço amostral correspondente (olhar probabilístico), este mesmo participante fez uso de listagem, registrando por escrito, lado a lado, cada ordem de leitura considerada, isto é, cada possibilidade levantada: Iracema, Senhora, Lucíola; Senhora, Iracema Lucíola;Lucíola, 
Iracema, Senhora; Senhora, Lucíola, Iracema; Luciola, Senhora, Iracema; Iracema, Lucíola, Senhora.A transcrição referente a tal problema é apresentada na Figura 8.

Figura 8. Exemplo de transcrição da entrevista clínica (P1)

Transcrição 4. Problema de espaço amostral de permutação (Teste 1), resolvido por P1 (Grupo 1). Listagem extensiva não sistemática: todas as possibilidades

[...] P1 lista rapidamente as seis ordens de leitura possíveis, demonstrando boa compreensão do invariante de ordem do problema.

Pesquisadora: "Quando você resolveu antes, fazendo a conta, a resposta foi nove, não foi? Você acha que são seis ou são nove ordens de leitura possíveis?"

P1: “[...] Seis. Não consigo escrever mais não... Tem mais não”

\section{Fonte: As autoras}

A partir da revisitação em questão, P1 conseguiu, assim, visualizar as possibilidades consideradas, esgotando-as (seis) e chegando à conclusão de que, inicialmente, ao utilizar a multiplicação inadequada, havia extrapolado o número de possibilidades existentes. Ajustou, portanto, sua resposta, chegando a um acerto total.

Em outro exemplo, P21 (Grupo 3), ao resolver o problema de combinação, enumerou oralmente possibilidades de escolha de trios a partir dos cinco primos de Sara. O participante baseou-sena disposição dos rapazes na ilustração para construir as três possibilidades consideradas, utilizando-se de nomes de primos que estavam lado a lado (André, Bruno e César; Bruno, César e Diogo; e César, Diogo e Eraldo).

Ao revisitar essa situação combinatória a partir do problema probabilístico de espaço amostral de combinação fazendo uso de listagem, P21 conseguiu indicar várias outras possibilidades válidas (Figura 9) mesmo não tendo chegado ao esgotamento (10 possibilidades). Nesse caso em específico, houve avanço de desempenho tanto quantitativo quanto qualitativo. Inicialmente P21 havia enumerado oralmente menos da metade das possibilidades (obtendo uma pontuação 0 nesse problema) e, pós revisitação via listagem, considerou mais da metade das possibilidades (obtendo pontuação 1). Esse participante demonstrou, também, abstração a partir do contexto explorado no problema, pois desprendeuse da disposição dos primos na ilustração para construir as possibilidades. 
Figura 9. Problema de espaço amostral de combinação (Teste 1), resolvido por P21 (Grupo 3). Listagem extensiva não sistemática: mais da metade das possibilidades

$$
\begin{aligned}
& \text { Andre, Brumo. Ceiror. } \\
& \text { Bruno Cesor. Diogo. } \\
& \text { Eraldo Amdre Cesor. } \\
& \text { Césor, Diogo André } \\
& \text { Brumo, Eraldo. Andre } \\
& \text { Andre, Diogo. Brumo } \\
& \text { Eraldo Diogo, Cesar. }
\end{aligned}
$$

Fonte: Dados da pesquisa

Como apontado anteriormente, as revisitações propostas no Teste 2 (no qual os problemas solicitavam, explicitamente, a listagem de possibilidades antes da apresentação dos problemas combinatórios) não foram tão proveitosas. Um ponto negativo nessa ordem articulação proposta (probabilidade $\rightarrow$ combinatória) relaciona-se ao fato de que grande parte dos estudantes apresentaram resistência em revisitar os problemas de espaço amostral. Os estudantes que utilizaram esse tipo de teste (metade da amostra de cada grupo) já haviam registrado por escrito (ou enumerado oralmente) cada uma das possibilidades consideradas e, muitas vezes, não possuíam um repertório de estratégias mais eficientes/refinadas para a resolução dos problemas e, portanto, decidiam manter a resposta obtida na tentativa anterior, sem fazer uma análise e revisão das construções.

A revisitação às possibilidades consideradas é, contudo, um rico momento para que sejam levantadas reflexões sobre os invariantes dos problemas e pode proporcionar ajustes às construções e levar à descoberta de novas possibilidades ou desconsideração de casos repetidos. Dessa forma, ao optar pela não revisitação aos problemas de espaço amostral, alguns estudantes desperdiçaram a oportunidade proporcionada pela articulação proposta.

Assim, mesmo que as revisitações propostas no Teste 2 tenham provocado resistência por parte de alguns participantes em revisar suas resoluções, proporcionou alguns avanços proveitosos para a melhoria do desempenho/desenvolvimento dos raciocínios foco do estudo.Nas Figuras 10 e 11 é ilustrado o caso de um estudante do Grupo 2, que ao utilizar a listagem no problema de espaço amostral de arranjo havia indicado o dobro das possibilidades existentes, revelando incompreensões do invariante de ordem. Na revisitação, revisando sua listagem, o participante eliminou as repetições, chegando ao acerto total. 
Figura 10. Problema de espaço amostral de arranjo (Teste 2), resolvido por P16 (Grupo 2). Listagem reduzida sistemática: Extrapolação das possibilidades

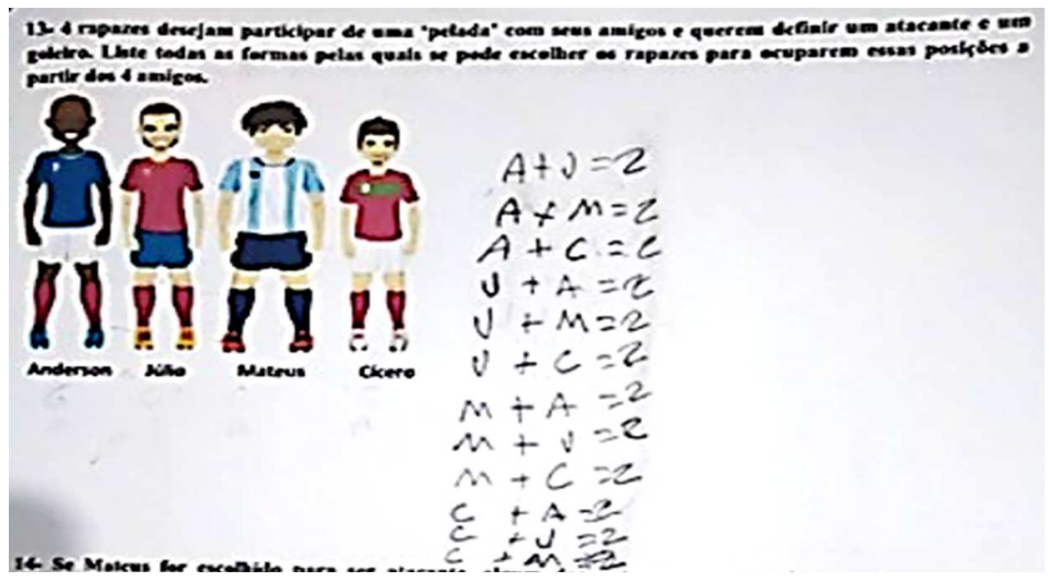

Fonte: Dados da pesquisa

Figura 11. Exemplo de transcrição da entrevista clínica (P16)

Transcrição 5. Problema de arranjo (Teste 2), resolvido por P16 (Grupo 2). Enumeração oral: todas as possibilidades

[...] P16 revisa a listagem feita anteriormente.

P16: "[...] Eu já tinha contado duas vezes, pra dizer que tinha trocado, mas ai eu escrevi de novo (risos)."

Pesquisadora: "Tem dupla repetida, então?"

P16: “'Tá' tudo repetido. Eu escrevi 24, mas 'é' só 12."

Não reescreveu a lista, apenas analisou a anteriormente construída e registrou o total (12).

Fonte: As autoras

Ao resolver o problema de espaço amostral de arranjo (Figura 10), P16 considerou corretamente o invariante de ordem do problema, indicando que cada dupla de rapazes poderia ser contada duas vezes, pois escolher Anderson para ocupar a vaga de atacante e Júlio para ocupar a vaga de goleiro é diferente de escolher Júlio para ser atacante e Anderson para ser goleiro, por exemplo. P16 representou tal possibilidade de inversão da dupla utilizando o número dois após cada par de rapazes listado. No entanto, posteriormente, P16 considerou essa inversão na própria listagem reduzida e indicou, assim, o dobro de possibilidades. No momento em que revisitou a listagem produzida, a partir da resolução do problema combinatório de arranjo, percebeu o erro e, oralmente, chegou ao acerto total.

É importante reforçar, portanto, que as revisitações propostas entre os problemas combinatórios e os problemas probabilísticos (construção de espaço amostral),em ambos os testes utilizados (diferentes ordens de articulação: combinatória $\rightarrow$ probabilidade e 
probabilidade $\rightarrow$ combinatória), proporcionaram importantes contribuições (principalmente qualitativas) que relacionam os raciocínios combinatório e probabilístico dos participantes da pesquisa.

Por fim, destaca-se que a pesquisa em questão explorou, ainda, articulações entre problemas combinatórios e outras demandas cognitivas ao amplo entendimento da probabilidade (Bryant \& Nunes, 2012). Os problemas que exploram a construção de espaços amostrais possuem uma relação mais direta com o raciocínio combinatório e são foco de outros estudos que ressaltam a importância da articulação entre combinatória e probabilidade a partir do uso de representações variadas (Kapon, Ron, Hershkowitz \& Dreyfus, 2015, Lockwood, 2012, Nunes, Bryant, Evans, Gottardis \& Terlektsi, 2014, Rubel, Lim, HallWieckert \& Sullivan, 2016), contudo, estes não foram os únicos a se relacionarem positivamente com os problemas combinatórios, como discutido no texto completo de dissertação (Lima, 2018) e em outros recortes publicados anteriormente (Lima \& Borba, 2018, 2019a, 2019b, 2020).

A seguir, são apresentadas algumas considerações acerca das análises e discussões presentes no presente texto.

\section{Algumas considerações}

Com a realização da pesquisa relatada constatou-se, corroborando estudos anteriores, um melhor desempenho no problema combinatório que explorou a situação de produto cartesiano e o menor desempenho no problema de combinação. No recorte apresentado no presente texto, teve-se por foco a articulação entre as diferentes situações combinatórias propostas e uma das demandas cognitivas ao amplo entendimento da probabilidade, relacionada à construção de espaços amostrais. A partir de tais revisitações em ambos os sentidos - Teste 1 (combinatória $\rightarrow$ probabilidade) e Teste 2 (probabilidade $\rightarrow$ combinatória) - foram observadas contribuições quantitativas e qualitativas nos desempenhos dos estudantes, bem como nas reflexões e falas que evidenciam seus raciocínios, revelando, ainda, a grande importância da realização das entrevistas clínicas para que fosse possível acompanhar de perto o processo de resolução dos problemas propostos.

No Teste 1, a construção de espaços amostrais (a partir de listagens variadas ou enumeração oral) se mostrou um momento rico de avaliação/revisão das estratégias utilizadas 
inicialmente e espontaneamente na solução dos problemas combinatórios propostos, auxiliando os estudantes no esgotamento ou aproximação ao número total de possibilidades, bem como de reflexão sobre os invariantes de ordem e de escolha desses problemas. No Teste 2, no qual a solicitação da construção de espaços amostrais foi feita antes da apresentação do problema combinatório, muitos estudantes apresentaram resistência em fazer revisitações aos problemas propostos, visto que não possuíam um repertório mais refinado de estratégias e respectivas representações para utilizar após a listagem/enumeração de possibilidades uma a uma. Tal decisão, no entanto, fez com que muitos estudantes perdessem a oportunidade de (re)analisar as construções feitas e invariantes considerados, o que poderia levá-los a avançar em seus desempenhos. Dessa maneira, a ordem de articulação presente no Teste 1 (situações combinatórias revisitadas via probabilidade - construção de espaços amostrais) se mostrou mais promissora na pesquisa em questão.

A escolarização formal influenciou, principalmente de maneira qualitativa, os desempenhos apresentados pelos estudantes pertencentes aos diferentes grupos. Essa influência esteve atrelada ao uso de diferentes estratégias e representações simbólicas, bem como à compreensão dos invariantes (de ordem e de escolha) dos diferentes tipos de problemas explorados.

Dado o destaque relacionado à listagem no presente trabalho, é necessário apontar que esta é uma importante estratégia/representação, intrinsecamente relacionada a problemas de natureza combinatória e probabilística nos quais o levantamento/quantificação de possibilidades é essencial, mas não é suficiente para a Educação Básica. Em momentos mais avançados da escolarização é necessário, tendo-se em vista o desenvolvimento dos raciocínios combinatório e probabilístico dos estudantes, que o ensino formal preze pela compreensão dos invariantes de diferentes situações que não dependa do uso de listagem/enumeração oral direta e que outras representações (mais refinadas e com capacidade de abstração) sejam conhecidas e dominadas. Dessa forma, os estudantes estarão aptos a resolver problemas mais complexos e com número maior de possibilidades envolvidas.

Assim, a instrução escolar deve proporcionar o desenvolvimento desses raciocínios (inclusive de maneira articulada), tendo por foco a ampliação do repertório de estratégias (como o princípio fundamental da contagem, fórmulas, etc.) e a sistematização das já utilizadas, visto que "as representações simbólicas têm justamente a vantagem de dar uma 
ajuda à resolução de um problema quando os dados são numerosos e a resposta à questão colocada exige várias etapas" (Vergnaud, 1996, p. 184). O uso de listagem (construção de espaço amostral) é um caminho promissor para a exploração dos invariantes dos problemas combinatórios, preparando os estudantes para o uso posterior do princípio fundamental da contagem e das fórmulas.

No intuito de dar continuidade ao estudo das relações entre combinatória e probabilidade, centro das discussões do presente artigo, encontra-se em andamento a pesquisa de doutoramento da primeira autora, sob orientação da segunda autora. Tal pesquisa busca aprofundar o olhar à combinatória, à probabilidade e às suas relações e possíveis articulações ao explorá-las em diferentes instâncias do currículo e tem por objetivo geral analisar articulações entre combinatória e probabilidade nos currículos prescritos e apresentados voltados aos anos finais do ensino fundamental, visando a construção de uma proposta que favoreça o ensino de ambas as áreas. Trabalhos referentes a resultados preliminares de diferentes etapas de tal estudo podem ser acessados no endereço www.geracaoufpe.blogspot.com, página de divulgação do Grupo de Estudos em Raciocínios Combinatório e Probabilístico, do qual ambas as autoras do presente texto são membros.

\section{Referências}

Azevedo, J. (2013). Alunos de anos iniciais construindo árvores de possibilidades: é melhor no papel ou no computador? Dissertação de Mestrado. Universidade Federal de Pernambuco, Recife.

Batanero, C., Godino, J. \& Navarro-Pelayo, V. (1996). Razonamiento combinatorio. Madrid: Síntesis.

Borba, R. (2010). O raciocínio combinatório na educação básica. Anais do Encontro Nacional de Educação Matemática $X$ ENEM. Disponível em: https://drive.google.com/file/d/0B3nOb_rG1DUhaHd2YVBKV1lRVm8/view

Borba, R. (2016). Antes cedo do que tarde: O aprendizado da combinatória no início da escolarização. Anais do Encontro de Combinatória, Estatística e Probabilidade dos Anos Iniciais Recife: Disponível em: https://drive.google.com/file/d/0ByUlyzknmdPLb0lBak04R1RnMWc/view

Bryant, P., Nunes, T. (2012). Children's understanding of probability: a literature review.Orford: UK: Nuffield Foundation

Carraher, T. (1998). O método clínico usando os exames de Piaget. São Paulo: Cortez.

Fernandes, J. A., Correia, P. \& Roa, R. (2010). Aquisição das operações combinatórias por alunos pré-universitários através de uma intervenção de ensino. Revista 
Latinoamericana de Investigación en Matemática Educativa, 13 (2), 215-242.

Kapon, S., Ron, G., Hershkowitz, R. \& Dreyfus, T. (2015). Perceiving permutations as distinct outcomes: the accommodation of a complex knowledge system. Educational Studies in Mathematics, 88(1), 43-64. https://doi.org/10.1007/s10649-014-9570-2.

Lima, E. (2018). Raciocínios combinatório e probabilístico na EJA: investigando relações. Dissertação de Mestrado. Universidade Federal de Pernambuco, Recife.

Lima, R. (2010). O raciocínio combinatório de alunos da educação de jovens e adultos: do início da escolarização até o ensino médio. Dissertação de Mestrado. Universidade Federal de Pernambuco. Recife.

Lima, E. \& Borba, R. (2017). A determinação de espaços amostrais na resolução de problemas combinatórios na EJA. Canoas: Anais do Congresso Internacional de Ensino da Matemática - $\quad$ VII CIEM. https://drive.google.com/file/d/1KdWJjxpegfDAQKPKhLR1BkUuDXi7DCAe/view

Lima, E. \& Borba, R. (2018). Relações entre o raciocínio combinatório e o raciocínio probabilístico na EJA. Revista Paranaense de Educação Matemática, 7(13), 33-60.

Lima, E. \& Borba, R. (2019a). Articulando os raciocínios combinatório e probabilístico a partir da resolução de problemas na EJA. Educação Matemática Pesquisa, 21(1), 136159. http://dx.doi.org/10.23925/1983-3156.2019v21i1p136-159

Lima, E. \& Borba, R. (2019b). A influência da escolarização na resolução de problemas combinatórios e probabilísticos: um estudo realizado na educação de jovens e adultos. Educação Matemática em Revista, 20(2), 134-146.

Lima, E. \& Borba, R. (2020). Investigando relações entre os raciocínios combinatório e probabilístico na educação de jovens e adultos. Acta Scientiae, 22(2), 134-149. https://doi.org/10.17648/acta.scientiae.5383

Lockwood, E. (2012). Counting using sets of outcomes. Mathematics Teaching in the Middle School, 18(3), 132-135.

MEC (1996). Lei de diretrizes e bases da educação nacional - Lei n. 9394/96. Brasília: MEC.

Morgado, A., Pitombeira de Carvalho, J., Pinto de Carvalho, P., \& Fernandez, P. (1991). Análise combinatória e probabilidade. Rio de Janeiro: Graftex.

Navarro-Pelayo, V., Batanero, C., \& Godino, J. (1996). Razonamiento combinatorio en alumnos de secundaria. Educación Matemática, 8(1), 26-39.

Nunes, T., Bryant, P., Evans, D., Gottardis, L., \& Terlektsi, M. (2014). The cognitive demands of understanding the sample space. ZDM. The International Journal on Mathematics Education, 46(3), 437-448.

Oliveira, M. K. (1999). Jovens e adultos como sujeitos de conhecimento e aprendizagem. ANPED. Revista Brasileira de Educação, 12, 59-73.

Pessoa, C. (2009). Quem dança com quem: o desenvolvimento do raciocínio combinatório do $2^{\circ}$ ano do Ensino Fundamental ao $3^{\circ}$ ano do Ensino Médio. Tese de Doutorado. Universidade Federal de Pernambuco, Recife.

Rubel, L., Lim, V., Hall-Wieckert, M., \& Sullivan, M. (2016). Teaching mathematics 
for spatial justice: an investigation of the lottery. Cognition and Instruction, 34(1), 126.

Sacristán, J. G. (2000). O currículo: Uma reflexão sobre a prática. Porto Alegre: Artmed.

Silva, R. (2016). É a moeda que diz, não é a gente que quer não: conhecimentos probabilísticos de crianças em situações de jogos. Dissertação de Mestrado. Universidade Federal de Pernambuco, Recife.

Souza, L., Souza, G., \& Silveira, M. (2019). Probabilidade no ensino médio: uma investigação ação em contraste com o currículo. Caminhos da Educação Matemática em Revista, 9(2), 205-222.

Vergnaud, G. (1986). Psicologia do desenvolvimento cognitivo e didática das matemáticas. Um exemplo: as estruturas aditivas. Análise Psicológica, 1, 75-90.

Vergnaud, G. (1996). A teoria dos campos conceptuais. In J. Brum (Org.),Didáctica das Matemáticas (pp. 155-191). Lisboa: Horizontes Pedagógicos,

Zwanch, K. (2019). A preliminary genetic decomposition of probabilistic independence. The Mathematics Educator, 28(1), 3-26.

\section{Autoras}

Ewellen Tenório de Lima

Professora de Matemática na Rede Estadual de Ensino da Paraíba. Licenciada em Matemática (Universidade de Pernambuco - UPE). Mestre em Educação Matemática e Tecnológica (Universidade Federal de Pernambuco - UFPE). Doutoranda em Educação Matemática e

Tecnológica (UFPE). Membro do Grupo de Estudos em Raciocínios Combinatório e Probabilístico - Geração. Linha de Investigação: Processos de ensino e de aprendizagem.

Email: ewellentlima@gmail.com

Rute Elizabete de Souza Rosa Borba

Professora da Universidade Federal de Pernambuco (UFPE). Mestre em Psicologia Cognitiva (UFPE). PhD (Oxford Brookes University). Líder do Grupo de Estudos em Raciocínios Combinatório e Probabilístico - Geração. Linha de Investigação: Processos de ensino e de aprendizagem. Email: resrborba@gmail.com 